\title{
Global analysis of gene expression dynamics within the marine microbial community during the VAHINE mesocosm experiment in the southwest Pacific
}

\author{
Ulrike Pfreundt ${ }^{1}$, Dina Spungin ${ }^{2}$, Sophie Bonnet ${ }^{3,4}$, Ilana Berman-Frank ${ }^{2}$, and Wolfgang R. Hess ${ }^{1}$ \\ ${ }^{1}$ Institute for Biology III, University of Freiburg, Freiburg, Germany \\ ${ }^{2}$ Mina and Everard Goodman Faculty of Life Sciences, Bar Ilan University, Ramat Gan, Israel \\ ${ }^{3}$ Aix Marseille Université, CNRS/INSU, Université de Toulon, IRD, Mediterranean Institute of Oceanography \\ (MIO), Marseille, France \\ ${ }^{4}$ Institut de Recherche pour le Développement, AMU/ CNRS/INSU, Université de Toulon, Mediterranean Institute \\ of Oceanography (MIO) Nouméa, New Caledonia, France
}

Correspondence to: Wolfgang R. Hess (wolfgang.hess@ biologie.uni-freiburg.de)

Received: 5 November 2015 - Published in Biogeosciences Discuss.: 23 February 2016

Revised: 21 June 2016 - Accepted: 4 July 2016 - Published: 19 July 2016

\begin{abstract}
Microbial gene expression was followed for 23 days within a mesocosm (M1) isolating $50 \mathrm{~m}^{3}$ of seawater and in the surrounding waters in the Nouméa lagoon, New Caledonia, in the southwest Pacific as part of the VAriability of vertical and tropHIc transfer of diazotroph derived $\mathrm{N}$ in the south wEst Pacific (VAHINE) experiment. The aim of VAHINE was to examine the fate of diazotroph-derived nitrogen (DDN) in a low-nutrient, low-chlorophyll ecosystem. On day 4 of the experiment, the mesocosm was fertilized with phosphate. In the lagoon, gene expression was dominated by the cyanobacterium Synechococcus, closely followed by Alphaproteobacteria. In contrast, drastic changes in the microbial community composition and transcriptional activity were triggered within the mesocosm within the first 4 days, with transcription bursts from different heterotrophic bacteria in rapid succession. The microbial composition and activity of the surrounding lagoon ecosystem appeared more stable, although following similar temporal trends as in M1. We detected significant gene expression from Chromerida in M1, as well as the Nouméa lagoon, suggesting these photoautotrophic alveolates were present in substantial numbers in the open water. Other groups contributing substantially to the metatranscriptome were affiliated with marine Euryarchaeota Candidatus Thalassoarchaea (inside and outside) and Myoviridae bacteriophages likely infecting Synechococcus, specifically inside M1. High transcript abun-
\end{abstract}

dances for ammonium transporters and glutamine synthetase in many different taxa (e.g., Pelagibacteraceae, Synechococcus, Prochlorococcus, and Rhodobacteraceae) was consistent with the known preference of most bacteria for this nitrogen source. In contrast, Alteromonadaceae highly expressed urease genes; Rhodobacteraceae and Prochlorococcus showed some urease expression, too. Nitrate reductase transcripts were detected on day 10 very prominently in Synechococcus and in Halomonadaceae. Alkaline phosphatase was expressed prominently only between days 12 and 23 in different organisms, suggesting that the microbial community was not limited by phosphate, even before the fertilization on day 4 , whereas the post-fertilization community was.

We observed high expression of the Synechococcus sqdB gene, only transiently lowered following phosphate fertilization. $S q d B$ encodes UDP-sulfoquinovose synthase, possibly enabling marine picocyanobacteria to minimize their phosphorus requirements by substitution of phospholipids with sulphur-containing glycerolipids. This result suggests a link between $s q d B$ expression and phosphate availability in situ.

Gene expression of diazotrophic cyanobacteria was mainly attributed to Trichodesmium and Richelia intracellularis (diatom-diazotroph association) in the Nouméa lagoon and initially in M1. UCYN-A (Candidatus Atelocyanobacterium) transcripts were the third most abundant and de- 
clined both inside and outside after day 4 , consistent with 16S- and nifH-based analyses. Transcripts related to the $E p$ ithemia turgida endosymbiont and Cyanothece ATCC 51142 increased during the second half of the experiment.

\section{Introduction}

In the study of natural marine microbial populations, it is of fundamental interest to identify the biota these populations consist of and to elucidate their transcriptional activities in response to biotic or abiotic changes in the environment. Metatranscriptomics gives insight into these processes at high functional and taxonomic resolution, as shown, e.g., in the analysis of a wide range of marine microbial populations (Frias-Lopez et al., 2008; Ganesh et al., 2015; Gifford et al., 2014; Hewson et al., 2010; Hilton et al., 2015; Jones et al., 2015; Moran et al., 2013; Pfreundt et al., 2014; Poretsky et al., 2009; Shi et al., 2009; Steglich et al., 2015; Wemheuer et al., 2015). Here, we report the results of a metatranscriptome analysis from the VAriability of vertical and tropHIc transfer of diazotroph derived $\mathrm{N}$ in the south wEst Pacific (VAHINE) mesocosm experiment, whose overarching objective was to examine the fate of diazotroph-derived nitrogen (DDN) in a low-nutrient, low-chlorophyll (LNLC) ecosystem (Bonnet et al., 2016b). In this experiment, three largescale $\left(\sim 50 \mathrm{~m}^{3}\right)$ mesocosms were deployed enclosing ambient oligotrophic water from the Nouméa (New Caledonia) lagoon in situ. To alleviate any potential phosphate limitation and stimulate the growth of diazotrophs, the mesocosms were fertilized on day 4 with $0.8 \mu \mathrm{mol} \mathrm{KH}_{2} \mathrm{PO}_{4}$ as a source of dissolved inorganic phosphorus (DIP). The mesocosms were sampled daily for 23 days and analyzed for carbon, nitrogen, and phosphorus pools and fluxes (Berthelot et al., 2015), the diazotroph community composition on the basis of nifH tag sequencing (Turk-Kubo et al., 2015), $\mathrm{N}_{2}$ fixation dynamics, and the fate of DDN in the ecosystem (Berthelot et al., 2015; Bonnet et al., 2016a; Knapp et al., 2015). Furthermore, the composition, succession, and productivity of the autotrophic and heterotrophic communities were studied (Leblanc et al., 2016; Pfreundt et al., 2016; Van Wambeke et al., 2016). During days $15-23$ of the VAHINE experiment, $\mathrm{N}_{2}$ fixation rates increased dramatically, reaching $>60 \mathrm{nmol} \mathrm{N} \mathrm{L}^{-1} \mathrm{~d}^{-1}$ (Bonnet et al., 2016a), which are among the highest rates reported for marine waters (Bonnet et al., 2016a; Luo et al., 2012). Based on the analysis of nifH sequences, $\mathrm{N}_{2}$-fixing cyanobacteria of the UCYN-C type were suggested to dominate the diazotroph community in the mesocosms at this time (Turk-Kubo et al., 2015). Evidence from ${ }^{15} \mathrm{~N}$ isotope labeling analyses indicated that the dominant source of nitrogen fueling export production shifted from subsurface nitrate assimilated prior to the start of the 23-day experiment to $\mathrm{N}_{2}$ fixation by the end (Knapp et al., 2015). To link these data to the actual specific activities of different microbial taxa, here we present the community-wide gene expression based on metatranscriptomic data from one representative mesocosm (M1). Throughout the course of the experiment (23 days), we sampled water from both M1 and the surrounding Nouméa lagoon every second day from the surface $(1 \mathrm{~m})$ and inferred the metatranscriptomes for the plankton fraction $(<1 \mathrm{~mm})$.

\section{Methods}

\subsection{Sampling, preparation of RNA and sequencing libraries}

Samples were collected in January 2013 every other day at 07:00 LT from mesocosm 1 (hereafter called M1) and from the Nouméa lagoon (outside the mesocosms) in $10 \mathrm{~L}$ carboys using a Teflon pump connected to PVC tubing. To ensure quick processing of samples, the carboys were immediately transferred to the inland laboratory setup on Amédée Island, located 1 nautical mile off the mesocosms. Samples for RNA were prefiltered through a $1 \mathrm{~mm}$ mesh to keep out larger eukaryotes and then filtered on $0.45 \mu \mathrm{m}$ polyethersulfone filters (Pall Supor). These filters were immediately immersed in RNA resuspension buffer (10 mM NaAc pH 5.2, $200 \mathrm{mM}$ $\mathrm{D}(+)$-sucrose, $100 \mathrm{mM} \mathrm{NaCl}, 5 \mathrm{mM}$ EDTA) and snap frozen in liquid nitrogen. Tubes with filters were vortexed, then agitated in a Precellys bead beater (Peqlab, Erlangen, Germany) $2 \times 15 \mathrm{~s}$ each at $6500 \mathrm{rpm}$ after adding $0.25 \mathrm{~mL}$ glass beads $(0.10-0.25 \mathrm{~mm}$, Retsch, Frimley, UK) and $1 \mathrm{~mL}$ PGTX (39.6 g phenole, $6.9 \mathrm{~mL}$ glycerol, $0.1 \mathrm{~g}$ 8-hydroxyquinoline, $0.58 \mathrm{~g}$ EDTA, $0.8 \mathrm{~g} \mathrm{NaAc}, 9.5 \mathrm{~g}$ guanidine thiocyanate, $4.6 \mathrm{~g}$ guanidine hydrochloride, $\mathrm{H}_{2} \mathrm{O}$ to $100 \mathrm{~mL}$; Pinto et al., 2009). We isolated RNA for metatranscriptomics and DNA for $16 \mathrm{~S}$ tag-based community analysis (Pfreundt et al., 2016) from the same samples by adding $0.7 \mathrm{~mL}$ chloroform, vigorous shaking, incubation at $24^{\circ} \mathrm{C}$ for $10 \mathrm{~min}$, and subsequent phase separation by centrifugation. RNA and DNA was retained in the aqueous phase, precipitated together and stored at $-80^{\circ} \mathrm{C}$ for further use.

The samples were treated by TurboDNase (Ambion, Darmstadt, Germany), purified with RNA Clean \& Concentrator columns (Zymo Research, Irvine, USA), followed by Ribozero (Illumina Inc., USA) treatment for the depletion of ribosomal RNAs. To remove the high amounts of tRNA from the rRNA depleted samples, these were purified further using the Agencourt RNAClean XP kit (Beckman Coulter Genomics). Then, first-strand cDNA synthesis was primed with an N6 randomized primer. After fragmentation, Illumina TruSeq sequencing adapters were ligated in a strandspecific manner to the 5' and 3' ends of the cDNA fragments, allowing the strand-specific PCR amplification of the cDNA with a proof-reading enzyme in 17-20 cycles, depending on yields. To secure that the origin of each sequence could be tracked after sequencing, hexameric TruSeq barcode sequences were used as part of the 3' TruSeq sequencing 
adapters. The cDNA samples were purified with the Agencourt AMPure XP kit (Beckman Coulter Genomics), quality controlled by capillary electrophoresis and sequenced by a commercial vendor (Vertis Biotechnologie AG, Germany) on an Illumina NextSeq 500 system using the paired-end $(2 \times 150 \mathrm{bp})$ setup. All raw reads can be downloaded from the NCBI Sequence Read Archive under the BioProject accession number PRJNA304389.

\subsection{Pretreatment and de-novo assembly of metatranscriptomic data}

Raw paired-end Illumina data in FASTQ format were pretreated as follows (read pairs were treated together in all steps to not produce singletons): adapters were removed and each read trimmed to a minimum Phred score of 20 using cutadapt. This left 386010015 pairs of good-quality raw reads for the 22 samples. Ribosomal RNA reads were removed using SortMeRNA (Kopylova et al., 2012). The resulting nonrRNA reads (corresponding to a total of 155022426 pairs of raw reads binned from all samples) were used as input for de-novo transcript assembly with Trinity (Haas et al., 2013) using digital normalization prior to assembly to even out kmer coverage and reduce the amount of input data. Remarkably, data reduction by digital normalization was only $\sim 35 \%$, hinting at a high complexity of the data set. This complexity is not surprising regarding that the sample pool contained transcripts from 3 weeks of experiment in two locations (mesocosm vs. lagoon), yet it also means that there will be a relatively large number of transcripts with very low sequencing coverage. This study thus misses the very rare transcripts in the analyzed community.

The transcript assembly led to 5594171 transcript contigs with an N50 of $285 \mathrm{nt}$, a median contig length of $264 \mathrm{nt}$, and an average of $326 \mathrm{nt}$. Transcript abundance estimation and normalization was done using scripts included in the Trinity package. Align_and_estimate_abundance.pl used bowtie (Langmead, 2010) to align all reads against all transcript contigs in paired-end mode, then ran RSEM (Li and Dewey, 2011) to estimate expected counts, TPM, and FPKM values for each transcript in each sample. Only paired-end read support was taken into account. The script Abundance_estimates_to_matrix.pl was modified slightly to create a matrix with RSEM expected counts and a TMM-normalized (trimmed mean of M-values normalization method) TPM matrix (the original script uses FPKM here) using the $\mathrm{R}$ package edgeR. The latter matrix was used to discard transcript contigs with very low support $(\operatorname{maxTPM}>=0.25$ and meanTPM $>=0.02)$. The remaining 3844358 transcript contigs were classified using the Diamond tool (Buchfink et al., 2015) with a blastX-like database search (BLOSUM62 scoring matrix, maximum $e$ value 0.001 , minimum identity $10 \%$, minimum bit score 50 ) against the NCBI nonredundant protein database from October 2015. Normalized TPM values for each transcript contig were added as a weight to the query ID in the Diamond tabular output samplewise with a custom script, creating one Diamond table per sample which served as input to Megan 5.11.3 (Huson and Weber, 2013). Megan is an interactive tool used here to explore the distribution of blast hits within the NCBI taxonomy and KEGG hierarchy. The parameters used to import the diamond output into Megan were minimum $e$ value 0.01 , minimum bit score 30 , LCA $5 \%$ (the transcript will be assigned to the last common ancestor of all hits with a bit score within $5 \%$ of the best hit), minimum complexity 0.3 .

During manual analysis of the top 100 transcript contigs according to their mean expression over all samples, we found 9 transcripts to be residual ribosomal RNA or internal transcribed spacer. These contigs were removed from the count and TPM matrices for all multivariate statistics analyses. Absence of these rRNA transcripts in the Diamond output was checked and verified.

\subsection{Sample clustering and multivariate analysis}

The matrix with expected counts for each transcript contig (see Sect. 2.2) was used as input for differential expression (DE) analysis with edgeR (Robinson et al., 2010) as implemented in the Trinity package script run_DE_analysis.pl for the set of samples taken from M1 and the Nouméa lagoon, respectively. The edgeR package can compute a DE analysis without true replicates by using a user-defined dispersion value, in this case 0.1 . We are aware that significance values are highly dependent on the chosen dispersion, and thus only considered transcripts with at least a 4-fold expression change for further DE analysis. The script analyze_diff_expr.pl was used (parameters $-\mathrm{P} 1 \mathrm{e}-3-\mathrm{C} 2$ ) to extract those transcripts that were at least 4 -fold differentially expressed at a significance of $\leq 0.001$ in any of the pairwise sample comparisons, followed by hierarchical clustering of samples and differentially expressed transcripts depending on normalized expression values $\left(\log _{2}(\mathrm{TPM}+1)\right)$. The resulting clustering dendrogram was cut using define_clusters_by_cutting_tree.pl at $20 \%$ of the tree height, producing subclusters of similarly responding transcripts.

Nonmetric multidimensional scaling (NMDS) was performed in $\mathrm{R}$ on the transposed matrix containing all 3844358 transcript contigs and their respective TMMnormalized TPM values. First, the matrix values were standardized to raw totals (sample totals) with the decostand function of the vegan package (Oksanen et al., 2015). Then, metaMDS was used for calculation of Bray-Curtis dissimilarity and the unconstrained ordination.

\subsection{Analysis of specific transcripts}

A list with genes of interest was created using the Integrated Microbial Genomes (IMG) system (Markowitz et al., 2015). 
First, 147 genomes close to bacteria and archaea found in the samples (based on 16S rRNA sequences; Pfreundt et al., 2016) were selected using "find genomes". Then, the "find genes" tool was used to find a gene of interest (for example nifH) in all the preselected genomes and the resulting genes added to the "gene cart". This was done for all genes of interest and the full gene list including $50 \mathrm{nt}$ upstream of each gene (possible 5' UTR) downloaded in FASTA format. Usearch_local (Edgar, 2010) was used to find all transcripts mapping to any of the genes in the list with a minimum query coverage and minimum identity of $60 \%$.

From the full Diamond output (Sect. 2.2), all matching transcripts together with their taxonomic and functional assignment were extracted and false positives discarded (i.e., transcripts that mapped to the list of specific genes but had a different Diamond hit). The top hit for each transcript was extracted, and the protein classifications manually curated to yield one common description per function (from different annotations for the same protein in different genomes). The TMM-normalized TPM counts were added to each transcript classification, as well as the full taxonomic lineage from NCBI taxonomy. These taxonomic lineages were curated manually to align taxonomic levels per entry.

The table was imported into $\mathrm{R}$, all counts per sample summed up for each combination of protein and family-level taxa, and a matrix created with samples as row names and combined protein and family description as column names. Heat maps were created separately for each protein group (e.g., rhodopsins or sulfolipid biosynthesis proteins), scaling all values to the group maximum.

\section{Results and discussion}

The metatranscriptomic data were analyzed following the strategy outlined in Fig. 1. We obtained taxonomic assignments for $37 \%$ of all assembled transcript contigs. This reflects the fact that the genes of complex marine microbial communities, especially from infrequently sampled ocean regimes like the southwest Pacific, are still insufficiently covered by current databases. The data with taxonomic assignments thus give an overview about the gene expression processes during this mesocosm experiment. With this study, we aimed at identifying global differences in expression patterns between the mesocosm and the lagoon, as well as between the different sampling time points within the mesocosm. We further explored the expression of marker genes for $\mathrm{N}$ and $\mathrm{P}$ metabolism, and light utilization in the different taxonomic groups.

\subsection{Transcripts cluster into distinct groups with similar expression patterns over time in M1 and the lagoon}

Gene expression changes roughly followed the timeline, within both M1 and the Nouméa lagoon, with some exceptions (Fig. 2). For the lagoon, samples from day 20 and 23 clustered together, the samples from day 10 to 18 formed a mid-time cluster, and those from day 2 to 8 an early cluster (Fig. 2b). In M1, the samples from day 6 to 10 and day 12 to 20 clustered together (Fig. 2a). Deviating from the timeline, the sample from day 2 was placed close to day 20, day 23 was separated from the late cluster, and day 4, exhibiting a prominent subcluster of transcripts upregulated only that day, was the furthest apart from all other samples (Fig. 2a, black brackets). Closer inspection of this subcluster containing several hundred different transcripts identified $>80 \%$ of them as Rhodobacteraceae transcripts, correlating well with a 5-fold increase of Rhodobacteraceae 16S tags (from 2.5 to $12.5 \%$ of the $16 \mathrm{~S}$ community) and a leap in bacterial production between T2 and T4 (Pfreundt et al., 2016). The observed transcripts were broadly distributed across metabolic pathways, reflecting a general increase of Rhodobacteraceae gene expression on day 4 . The aberrant clustering of the two earliest samples in M1 (before the DIP spike) and the tight clustering of those following the DIP spike (day 6-10) suggest an impact of the confinement within the mesocosm and of phosphate supplementation on gene expression.

Unconstrained ordination using nonmetric multidimensional scaling (NMDS) confirmed the similar temporal distribution of samples from the Nouméa lagoon and M1 (Fig. 3). Yet, the samples from M1 showed a much higher variance and were more dispersed than those from the lagoon (Fig. 3). Thus, the gene expression profiles within the mesocosm were more diverse than in the lagoon waters. The comparison of the whole data set against the KEGG database (Kanehisa et al., 2014) showed a major difference between M1 and the lagoon samples only in the category energy metabolism, and its subcategories photosynthesis and antenna proteins. These categories comprised 22-36, 8-16, and 2.7-7.5\% in the lagoon, respectively, and were in M1 (excluding day 23) constantly below 22, 7, and $4 \%$, respectively (the Supplement Figs. S1 and S2). This lower contribution of energy-related functions in M1 was detectable already at the earliest time point (day 2). Furthermore, diverging dynamics in the microbial community composition and transcriptional activity were triggered in M1 already within the first $48 \mathrm{~h}$ (before day 2 was sampled), indicated by the large distance between M1 and lagoon samples on day 2 (Fig. 3). The early timing of this effect already on day 2 suggests a rapid remodeling of the microbial community's gene expression upon confinement within the mesocosm. In addition, the DIP spike on the evening of day 4 subsequently triggered distinct ecological successions in M1. The patterns we observed here are close to the three temporal phases defined for the VAHINE experiment based on biogeochemical flux measurements (Bon- 


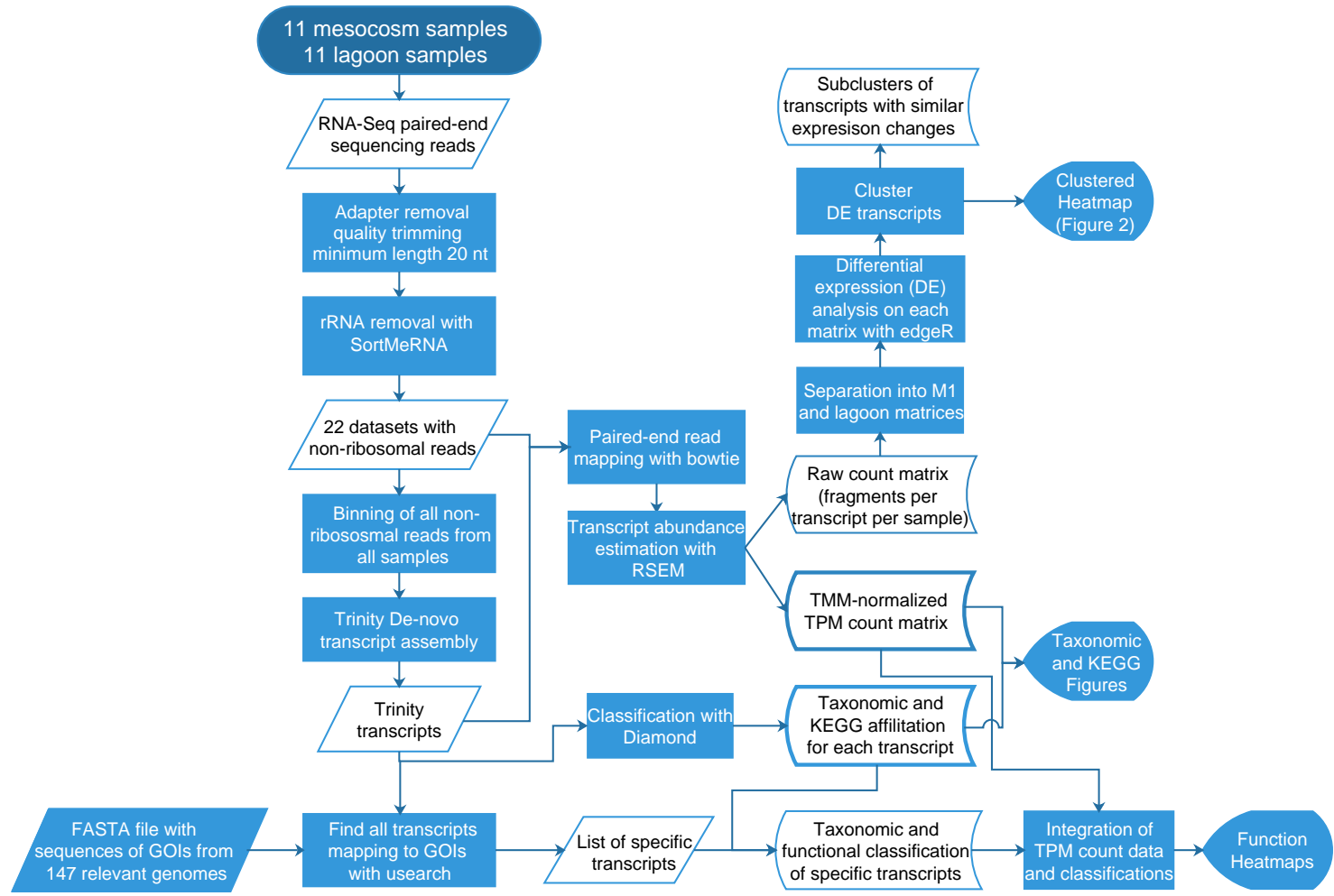

Figure 1. Flowchart describing the major steps in the bioinformatics workflow. Preprocessing of RNA-Seq reads was done separately for each data set, leading to 22 data sets of nonribosomal paired-end reads. These were binned and used as input for de-novo assembly of transcripts. The nonribosomal reads were mapped back onto the assembled transcripts with bowtie (Langmead, 2010) to infer each transcripts abundance in each sample using RSEM (Li and Dewey, 2011). Raw abundances were used for differential expression (DE) analysis and cluster analysis with edgeR on the M1 and lagoon count matrices separately, to find transcripts which changed significantly over time. To enable direct in-between sample comparison of transcript abundances, raw abundances were converted to TPM (transcripts per kilobase million) and TMM normalized (trimmed mean of $\mathrm{M}$ values) in RSEM, creating the final count matrix used for all figures showing transcript abundances. Classifications for these transcripts were generated using Diamond (Buchfink et al., 2015) against the RefSeq protein database. Further, a manually curated list with specific genes involved in N and P metabolism, as well as light utilization (genes of interest, GOIs) was used to extract the corresponding transcripts, but final classifications were inferred from the Diamond output. This information was used to produce the integrated function-per-taxon heat maps.

net et al., 2016a) and nifH amplicon analysis (Turk-Kubo et al., 2015). These were defined as follows (Bonnet et al., 2016a). Days 1-4 (phase P0): before the DIP fertilization, $\mathrm{P}$ deplete. Days 5-14 (P1): P availability, dominance of diatom-diazotroph associations. Days 15-23 (P2): decreasing $\mathrm{P}$ availability, slightly higher temperature, increasing $\mathrm{N}_{2}$ fixation and a dominance of UCYN-C diazotrophs in the mesocosms and increase of primary production (PP) inside and outside the mesocosms.

In the following sections, we refer to $\mathrm{P} 0, \mathrm{P} 1$, or $\mathrm{P} 2$ to describe trends and changes in gene expression when appropriate.

\subsection{Succession of gene expression inside mesocosm 1 and in the Nouméa lagoon}

\subsubsection{Active taxonomic groups differ between $\mathrm{M} 1$ and the lagoon}

The most striking difference between M1 and Nouméa lagoon samples was the 2- to 3-fold dominance of Oscillatoriophycideae transcripts over all other taxa in the lagoon over the full time of the experiment, but not in M1 (Figs. S3a, S4a). Gene expression within the Oscillatoriophycideae was mostly attributed to Synechococcus, with a substantial share of transcript reads in M1 and the lagoon coming from cyanobacteria closely related to Synechococcus CC9605, a strain representative of clade II within the picophytoplankton subcluster 5.1A (Dufresne et al., 2008) and Synechococcus RS9916, a representative of clade IX within picophytoplankton subcluster 5.1B (Scanlan et al., 

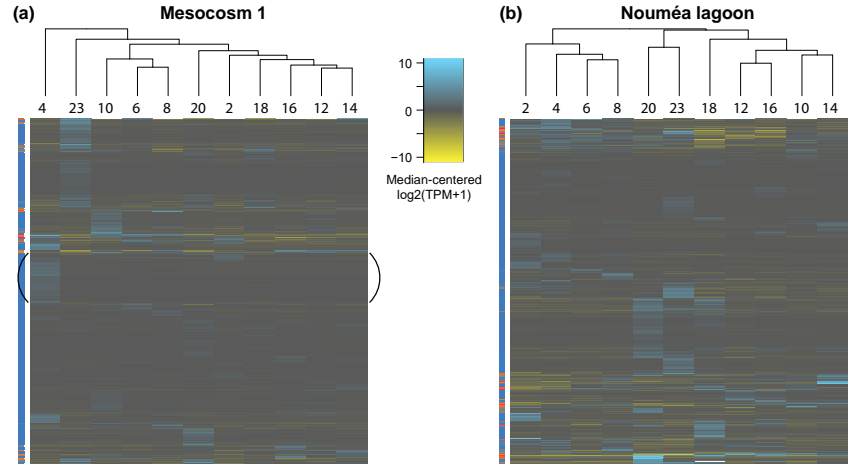

Figure 2. Heat map showing the expression (median-centered $\log _{2}(\mathrm{TPM}+1)$ ) of all significantly differentially expressed transcripts in all samples taken from mesocosm M1 (a) and outside (b). Clustering of samples and transcripts was done using Euclidean distance measures followed by average agglomerative clustering (hclust $($ method $=$ average)). Note that in (a) samples T2 and T4 cluster far away from the other samples. These were taken before the phosphate spike. In M1, T4 is distinguished by a large cluster of genes upregulated at that time point, most of which belong to the Rhodobacteraceae family. The general clustering along the timeline is evident inside and outside of M1.

2009; Figs. S3d, S4d). Clade II Synechococcus is typical for oligotrophic tropical or subtropical waters offshore or at the continent shelf, between $30^{\circ} \mathrm{N}$ and $30^{\circ} \mathrm{S}$ (Scanlan et al., 2009). Contrary to the Nouméa lagoon, Oscillatoriophycideae transcripts were lower than transcripts from Alphaand Gammaproteobacteria in M1 during phases P0 and P1 and only gained a similar level as in the lagoon in P2. We detected substantially higher gene expression from viruses in M1 compared to the Nouméa lagoon (Fig. 4). These were assigned mainly to Myoviridae such as S.SM2, S.SSM7, and other cyanophages of the T4-like group, which, based on their known host association (Frank et al., 2013; Ma et al., 2014), suggest a viral component acting on the Synechococcus fraction in the mesocosm. A burst of cyanophages might have contributed to the observed low numbers and activity of Synechococcus in M1 compared to the lagoon during P0 and P1 (Fig. 4). The recovery of Synechococcus populations in M1 during P2 mirrors the increase in the energy and photosynthesis-related functional categories (Figs. S1, S2) and in Synechococcus 16S tag abundance and cell counts (Leblanc et al., 2016; Pfreundt et al., 2016).

Owing to the initial decay of Synechococcus in M1, Alphaproteobacteria, mainly Rhodobacteraceae, SAR11, and SAR116, dominated the metatranscriptome during P0 (Fig. 4). Gammaproteobacterial transcripts increased at the beginning of $\mathrm{P} 1$, reaching similar levels as those of Alphaproteobacteria, and dropped again towards the end of P1, when the Synechococcus population started recovering (Fig. S3c). This suggests that the predominant Gammaproteobacteria profited from the organic matter released during

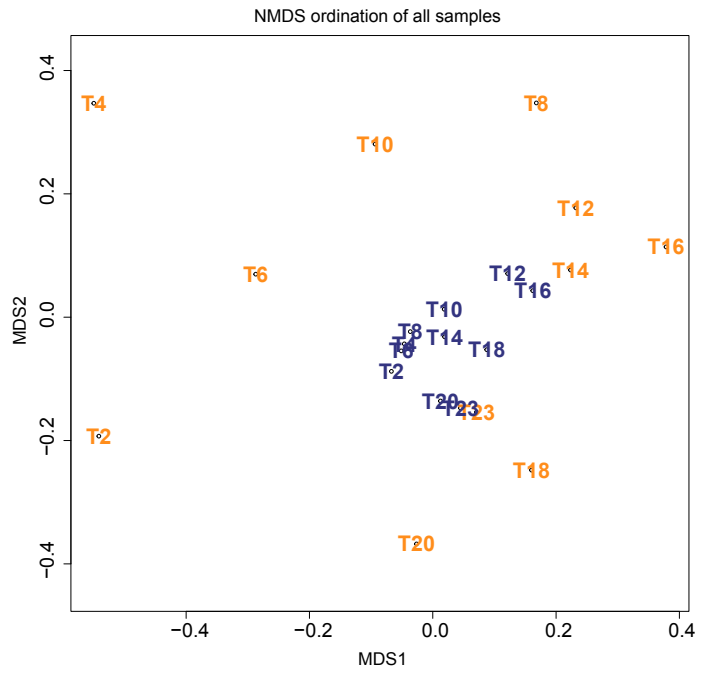

Figure 3. NMDS ordination of samples on the basis of TPM counts (transcripts per million sequenced transcripts). Outside samples are blue, samples from M1 are orange. Note that samples from M1 are more dispersed in the plot, thus transcription profiles are more diverse than outside. This might be due to the DIP fertilization creating a distinct ecological succession in M1.

bacterial decay. During P2, characterized by an abundant and very active Synechococcus population, Alphaproteobacteria gene expression increased again. Among these, only SAR11 transcripts decreased by about $75 \%$.

Unexpectedly, over 3 weeks, the temporal pattern of SAR11 transcription appeared tightly coordinated with that of SAR86 Gammaproteobacteria (Figs. S3, S4). We tested pairwise correlations of alpha- and gammaproteobacterial groups and found that SAR11 and SAR86 transcript accumulation were highly correlated in M1 and the Nouméa lagoon (Fig. S6, Pearson correlation: 0.88/0.96, Spearman rank correlation: $0.80 / 0.98$ for M1 and lagoon, respectively). This matches recent observations in both coastal and pelagic ecosystems for coupling of SAR11 and SAR86 gene expression throughout a diel cycle, suggesting specific biological interactions between these two groups (Aylward et al., 2015). The fact that we now see this correlation over 3 weeks in two replicate experiments (M1 and Nouméa lagoon sampling) strengthens this hypothesis substantially. On the other hand, transcriptional activity was decoupled from 16S-based abundance estimates for both clades (Pfreundt et al., 2016). Decoupling of specific activity and cell abundance has been noted before for SAR11, with specific activity being lower than cell abundance in the North Pacific (Hunt et al., 2013). In microcosm experiments, proteorhodopsin transcripts increased under continuous light while gene abundance decreased (Lami et al., 2009). No such information is available for SAR86 in the literature, and the reasons for this decoupling remain elusive. We found no hint in the transcriptional profile that could explain the burst in SAR11 16S tags in M1 

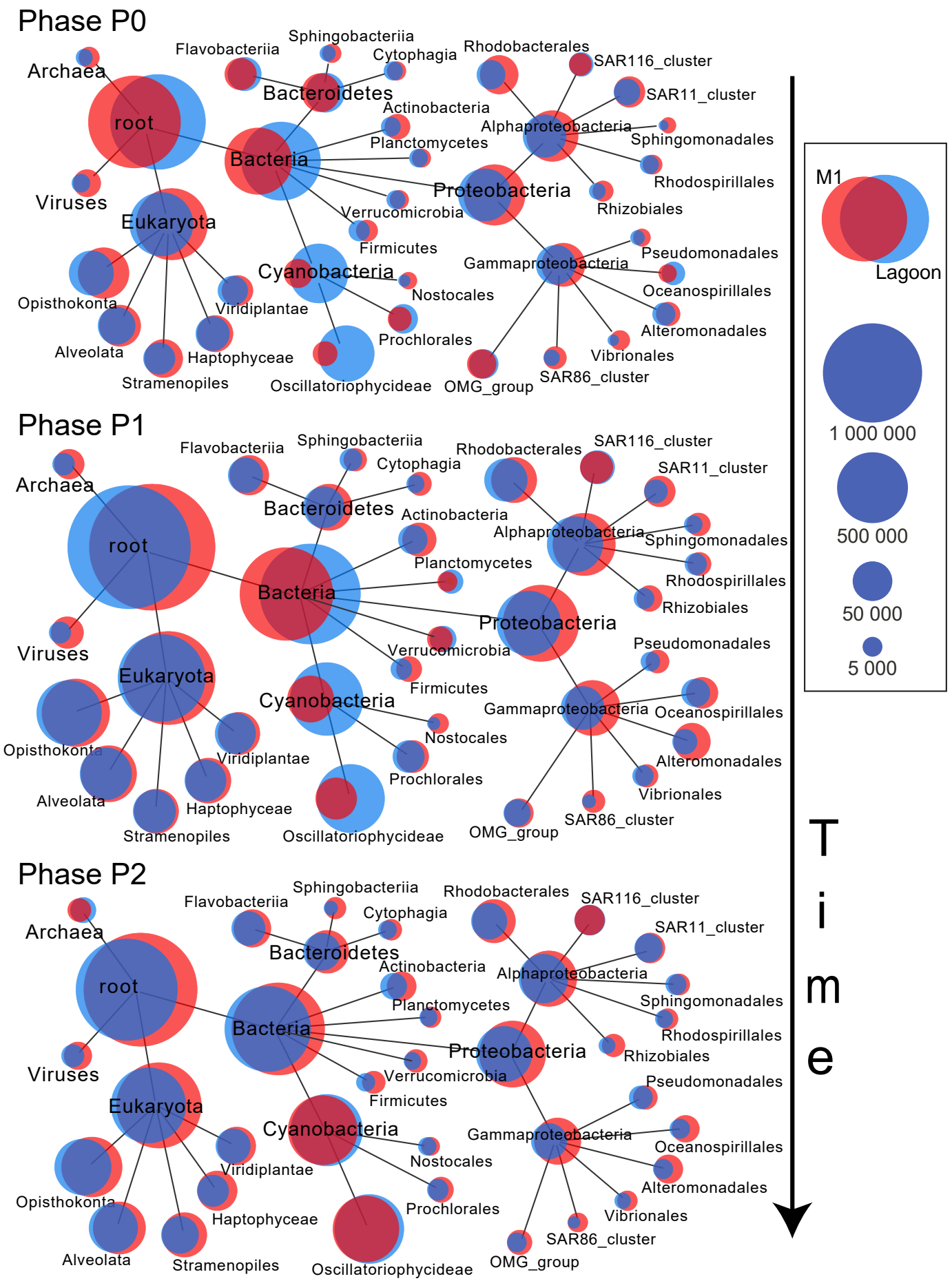

Figure 4. Comparison of the taxonomic affiliation of mRNA transcripts from M1 and the lagoon in the three chronological phases P0, P1, and P2, visualized with CoVennTree (Lott et al., 2015). Normalized transcript abundances (TMM-normalized TPM), displayed as the node area, were summed up per phase as follows. P0: day 2-day 4, P1: day 6-day 14, P2: day 16-day 23. The different sizes of the root nodes occur because different transcripts with differing total read abundances may be classifiable in the different data sets, and the data set normalization included all transcripts (also nonclassifiable). The overlap of the red (M1) and blue (lagoon) circles denotes the amount of transcripts present in both locations during the respective phase. The diagrams were reduced to show only major nodes and thus raise no claim to completeness. Yet, each node contains the information from all its children nodes, also those not shown. Archaea are scarcely represented in the current RefSeq protein database, thus their transcript abundances are underestimated here. 
on day 8 (from 8 to $26 \%$ ) and in the lagoon on day 16 (from 5 to $28 \%$; Pfreundt et al., 2016).

\subsubsection{Gene expression of oligotrophic marine Gammaproteobacteria (OMG) and Alteromonadaceae}

A closer look into gammaproteobacterial activities (Figs. S3c, S4c) revealed a dominant pool of transcripts from the oligotrophic marine Gammaproteobacteria (OMG) group (Cho and Giovannoni, 2004; Spring et al., 2013) and Alteromonadaceae, both in M1 and the lagoon. The temporal dynamics of the OMG group transcripts were very similar in both locations. The relatively high activity detected for OMG bacteria (similar to SAR11 activity) indicated that these aerobic anoxygenic photoheterotrophs (Spring et al., 2013) could thrive well both in M1 and the Nouméa lagoon at the start of the experiment. However, transcript accumulation declined constantly by 70-90\% until P2, then increased again during P2 until the end of the experiment, concurrent with Synechococcus activity and abundance. This pattern clearly decouples OMG group activity from phosphate availability, which was much higher in M1 than in the lagoon during $\mathrm{P} 1$, and also from the identity of the dominant diazotroph, which differed markedly between M1 and the lagoon in P2 (Turk-Kubo et al., 2015).

Alteromonadaceae-related transcript accumulation increased > 2.5-fold in M1 in the first half of phase P1, replacing the initially dominating OMG and SAR86 as the most active Gammaproteobacteria, but dropping to initial values in the second half of P1 (Fig. S3c). A burst in Alteromonas was previously reported as a confinement effect when a marine mixed microbial population was enclosed in mesocosms of smaller volumes (Schäfer et al., 2000). Immediately following the increase of Alteromonadaceae-related transcripts and reaching similar abundances, Idiomarinaceae transcripts increased 9-fold (Fig. S3c). This group of organotrophs is phylogenetically related to Alteromonadaceae. Gammaproteobacteria such as the Alteromonadaceae occur usually at rather low abundances in oligotrophic systems, but due to their copiotrophic metabolism (Ivars-Martinez et al., 2008; López-Pérez et al., 2012) increase in numbers and activity under eutrophic conditions or when particulate organic matter becomes available (García-Martínez et al., 2002; IvarsMartinez et al., 2008). The fact that bacterial production (measured by ${ }^{3} \mathrm{H}$-leucine assimilation) in M1 was not limited by phosphate (Van Wambeke et al., 2016) suggests that the DIP spike in the evening of day 4 was not responsible for these observations, but rather that both, Alteromonadaceae and Idiomarinaceae, reacted to nutrients released after the Rhodobacteraceae bloom on day 4 and the possibly virus-induced lysis of Synechococcus. Idiomarinaceae and Alteromonadaceae were transcriptionally very active compared to their 16S tag-based abundance estimates (Pfreundt et al., 2016), pointing at a tight regulation of their metabolic activities as a response to the appearance of suitable energy and nutrient sources.

\subsubsection{Subdominant gene expression: Microalgae, Flavobacteria, and Spirotricha}

Other groups following the dominant classes Oscillatoriophycideae, Alpha-, and Gammaproteobacteria regarding transcript abundance in M1 and in the Nouméa lagoon were Flavobacteria, and the eukaryotic Haptophyceae (Prymnesiophyceae), Chromerida and Spirotrichea (Figs. S3a and S5b, respectively). Chromerida are photoautotrophic alveolates and closely related to apicomplexan parasites. Chromerida have been isolated only from stony corals in Australian waters thus far (Moore et al., 2008; Oborník et al., 2012). Our finding of significant gene expression from Chromerida in samples from M1 (Fig. S3a), as well as the Nouméa lagoon (Fig. S5b) indicates they were present in substantial numbers in the open water. These findings are consistent with the predicted wider distribution, higher functional, and taxonomic diversity of chromerid algae (Oborník et al., 2012).

\subsubsection{Gene expression from nitrogen-fixing cyanobacteria}

We specifically examined the gene expression patterns of diazotrophic cyanobacteria (Fig. 5) and compared them with parallel analyses of nifH amplicon sequences (Turk-Kubo et al., 2015), whereas heterotrophic diazotrophs were orders of magnitude less abundant (Pfreundt et al., 2016; TurkKubo et al., 2015) and were not considered further. The nifH amplicon analyses demonstrated a shift in M1 from a diazotroph community composed primarily of Richelia (diatom-diazotroph associations, DDAs, Foster et al., 2011) and Trichodesmium during P0 and P1 (days 2-14) to approximately equal contributions from UCYN-C (unicellular $\mathrm{N}_{2}$ fixing cyanobacteria type $\mathrm{C}$ ) and Richelia in phase P2 (days 15-23, Turk-Kubo et al., 2015). This shift was not observed outside. Consistent with these findings in M1, we also measured dominant gene expression from Trichodesmium and Richelia spp. until day 14, and Candidatus Atelocyanobacterium thalassa (UCYN-A) until day 8, and an increase in transcripts mapping to the Epithemia turgida endosymbiont and Cyanothece sp. ATCC51142 (Fig. 5a), classified within the UCYN-C nifH group (Nakayama et al., 2014), in P2. The temporal dynamics of gene expression for Richelia spp. and Trichodesmium in M1 differed with Trichodesmium gene expression declining by $>97 \%$ from initiation of the experiment to day 12, while the gene expression from Richelia species was stable until day 10 and then declined by $~ 90 \%$ until day 16 (Fig. 5a). Except for the high (in relation to other diazotrophs) Trichodesmium transcript abundances on days 2 and 4; diazotroph transcript abundances matched well the nifH-gene-based reports (Turk-Kubo et al., 2015). Results 
(a) Transcript abundance of diazotrophs in mesocosm M1

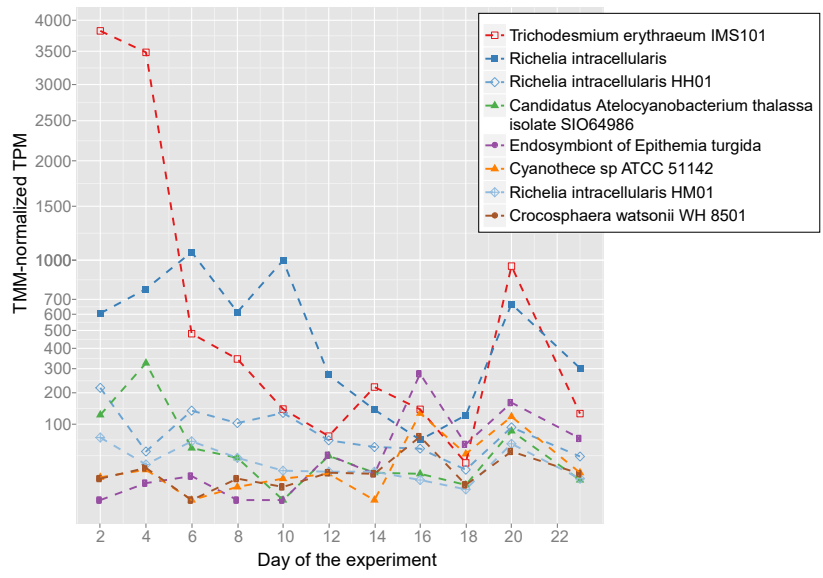

(b) Transcript abundance of diazotrophs in the Nouméa lagoon

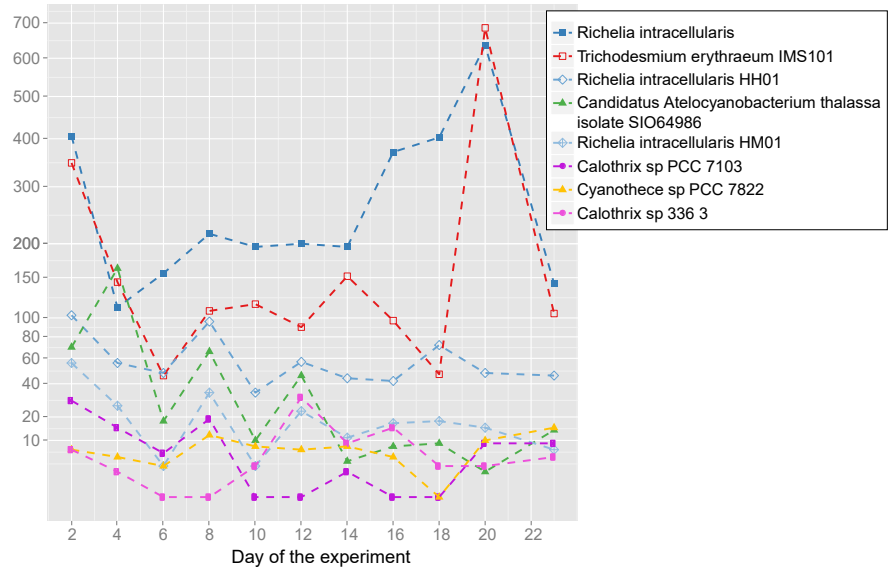

Figure 5. Gene expression in putative diazotrophic cyanobacteria inside M1 and in the Nouméa lagoon. Note the square-root scale for both plots and the generally higher transcript abundances inside M1. Transcriptional activity is presented in TPM (transcripts per million transcripts sequenced), normalized in between samples by TMM normalization (edgeR). Thus, plots can be directly compared, but values are relative.

are also consistent for the lagoon samples, where transcripts from Trichodesmium, Richelia and UCYN-A dominated the diazotroph transcript pool throughout the experiment and no UCYN-C transcripts were observed (Fig. 5b). Again, the high relative Trichodesmium transcript abundances between days 2 and 12 were not mirrored by $n i f H$-gene counts, while the rest were (Turk-Kubo et al., 2015). Notably, Trichodesmium transcripts were 1 order of magnitude lower in the lagoon than in M1.

\subsection{Specific analysis of relevant transcripts}

The 100 most highly expressed nonribosomal transcripts, as identified by highest mean expression in all samples, are presented in the Supplementary Table S1. Of them, 24 could not be classified with the NCBI nucleotide or protein databases and remain unknown. The most abundant transcript overall, both inside M1 and outside, was the non-protein-coding RNA (ncRNA) Yfr103, discussed in Sect. 3.4. All classified transcripts on the top 9 ranks plus 28 additional transcripts in M1 were related to Synechococcus and encoded mainly photosynthetic proteins or represented ncRNAs. The transcripts following on ranks $10-12$ in M1 plus five additional transcripts were affiliated with the recently defined new class of marine Euryarchaeota Candidatus Thalassoarchaea (Martin-Cuadrado et al., 2015), consistent with their detection by $16 \mathrm{~S}$ analysis (Pfreundt et al., 2016). Other top expressed transcripts were $r n p B$ from various bacteria, one tmRNA, and transcripts originating from the Rhodobacteraceae solely due to their expression peak on day 4 . We also detected three different abundant antisense RNAs (asRNAs), among them one transcribed from the complementary strand of Synechococcus gene Syncc8109_1164, encoding a hypothetical protein.

\subsubsection{Gene expression relevant for nitrogen assimilation}

To investigate gene-specific expression patterns, we analyzed genes of interest (GOIs) from specific genera. Transcripts mapping to the respective genes from different organisms were extracted, searched against NCBI's nonredundant protein database, and the hits were manually curated. This analysis was only performed for the M1 samples.

Genes indicative of different nitrogen utilization strategies are shown in Fig. 6. The selected GOIs were related to nitrogen fixation, nitrate and nitrite reduction, the uptake and assimilation of ammonia (transporter AmtA and glutamine synthetase, $g \ln A$ gene product), and the assimilation of urea. Signal transducer PII $(g \ln B$ gene product) and NtcA (nitrogen control transcription factor) were chosen as examples for the most important regulatory factors (Forchhammer, 2008; Huergo et al., 2013; Lindell and Post, 2001).

For Trichodesmium and UCYN-A (Candidatus Atelocyanobacterium), the core genes of the nitrogenase enzyme nifHDK were maximally expressed around day 4, while UCYN-C and Chromatiaceae (Gammaproteobacteria) nif expression peaked on day 20 (Fig. 6, nitrogen fixation). As nitrogenase gene expression and activity is under diel control, the expression patterns we obtained can only represent diazotrophs that fix $\mathrm{N}_{2}$ during the light hours because we sampled in the morning. The nifH phylogeny places the endosymbiotic diazotroph of Rhopalodia gibba within the UCYN-C group, but in contrast to other UCYN-C, these endosymbionts were shown to fix $\mathrm{N}_{2}$ in the light (Prechtl et al., 2004), explaining why our analysis captured their nif transcripts, but none mapping to Cyanothece sp. ATCC 51142. The nifH and nifD protein sequences of the R. gibba en- 


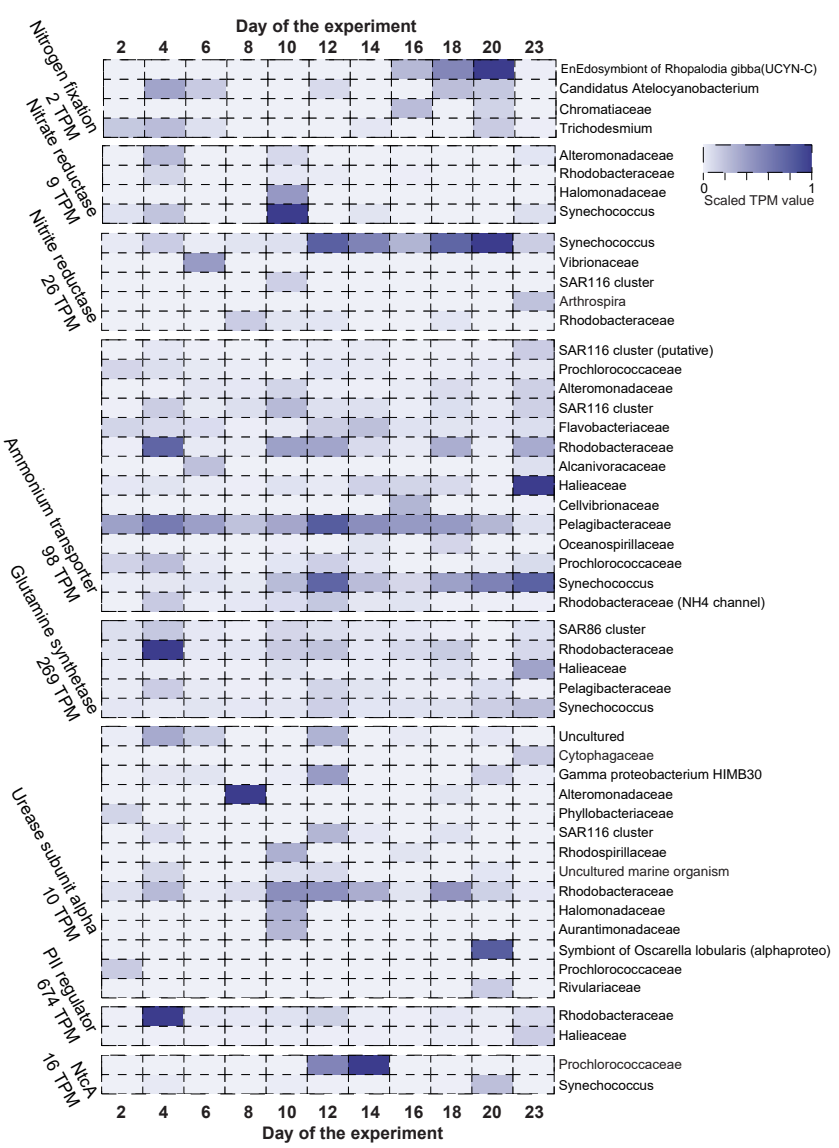

Figure 6. Expression of selected genes indicative for different nitrogen acquisition strategies. TPM counts were summed per taxonomic family, the names of which are denoted to the right of each line. The maximum TPM value for each group is written below the name of that group. For plotting, values were scaled within each functional group, but not for each line, resulting in the maximum color density always representing the maximum TPM. After the name, in brackets, is additional annotation information, if deviant from the name of the functional group. The nifHDK transcript counts were summarized for each taxon to avoid possible classification biases due to multicistronic transcripts (i.e., a multicistronic transcript might be classified as either of these depending on the best BLAST match).

dosymbiont and of Epithemia turgida, for which we report substantial gene expression in Sect. 3.2.4, are $98 \%$ identical, making it likely that the partial nif transcript contigs reported here could not be assigned unambiguously and probably belong to the Epithemia turgida symbiont or a close relative of both. Our data correlate with nifH gene abundance measured by Turk-Kubo et al. (2015). Note, that nif transcripts were generally very rare in this analysis (at maximum 2 TPM), making it very likely that below a certain expression threshold, a transcript was not sequenced at all.

Most other bacteria require ammonia, nitrate, or organic nitrogen sources such as urea, with ammonia being the energetically most favorable source. The importance of am- monia was underscored by expression of the respective uptake systems in many different taxa over long periods of the experiment and expression of glutamine synthetase (GS) (Fig. 6, ammonium transporter and glutamine synthetase), the enzyme forming the central point of entry for the newly assimilated nitrogen into the metabolism. Ammonia transporters (AMT) were highly expressed in the Pelagibacteraceae, throughout days 2-20, in Synechococcus from day 10 to the end, and in Rhodobacteraceae on day 4 (coinciding with maximum GS expression, and the global gene expression peak in this group, Fig. S3c). For the Halieaceae (OM60(NOR5) clade), the dominant family within the OMG group, AMT and GS expression did not coincide with their general expression peaks on day 2. Instead, these genes as well as the signal transducer PII were mainly expressed on day 23, indicating that Halieaceae were nitrogen limited toward the end of the experiment. Alteromonadaceae did not express either of these genes maximally on day 8 , when they were reaching their highest abundance and transcription. Instead, they expressed urease, constituting the highest measured urease expression in the whole experiment (Fig. 6, urease subunit alpha). Urea can serve as an alternative nitrogen source and is metabolized into ammonia. A shift towards urea utilization was also seen in Rhodobacteraceae. While most of the N-utilization transcripts analyzed here peaked on day 4 (coinciding with general expression and abundance peaks), urease expression in this group was highest from day 10 to 14 (Fig. 6, urease subunit alpha). Further, Prochlorococcus but not Synechococcus expressed urease, and both expressed ammonium transporters.

Nitrate reductase expression was detected on day 10 mainly in Synechococcus and in the Halomonadaceae. It is not clear why this gene is so strongly expressed on that single day, especially as nitrite reductase expression in Synechococcus was detectable over a longer period, from day 12 to day 20 (Fig. 6, nitrate reductase and nitrite reductase). Other taxa showed substantial nitrite reductase expression only on day 6 (Vibrionaceae), day 2 and days 14-16 (Rhodobacteraceae), or day 10 (SAR116).

The expression of the NtcA transcription factor itself can be an indicator for the nitrogen status, especially in marine picocyanobacteria (Lindell and Post, 2001; Tolonen et al., 2006). Therefore, the clear peaks for NtcA expression in Prochlorococcus on days 12-14, but in Synechococcus on day 20 indicate their divergent relative nitrogen demands (Fig. 6, NtcA). Noteworthy, ntcA expression was much stronger in Prochlorococcus than in Synechococcus compared to their 16S-based abundances and Prochlorococcus did not express it during its first abundance peak on day 6 , but only during the second one (Van Wambeke et al., 2016). The same was true for Synechococcus and its first abundance peak on day 12. 


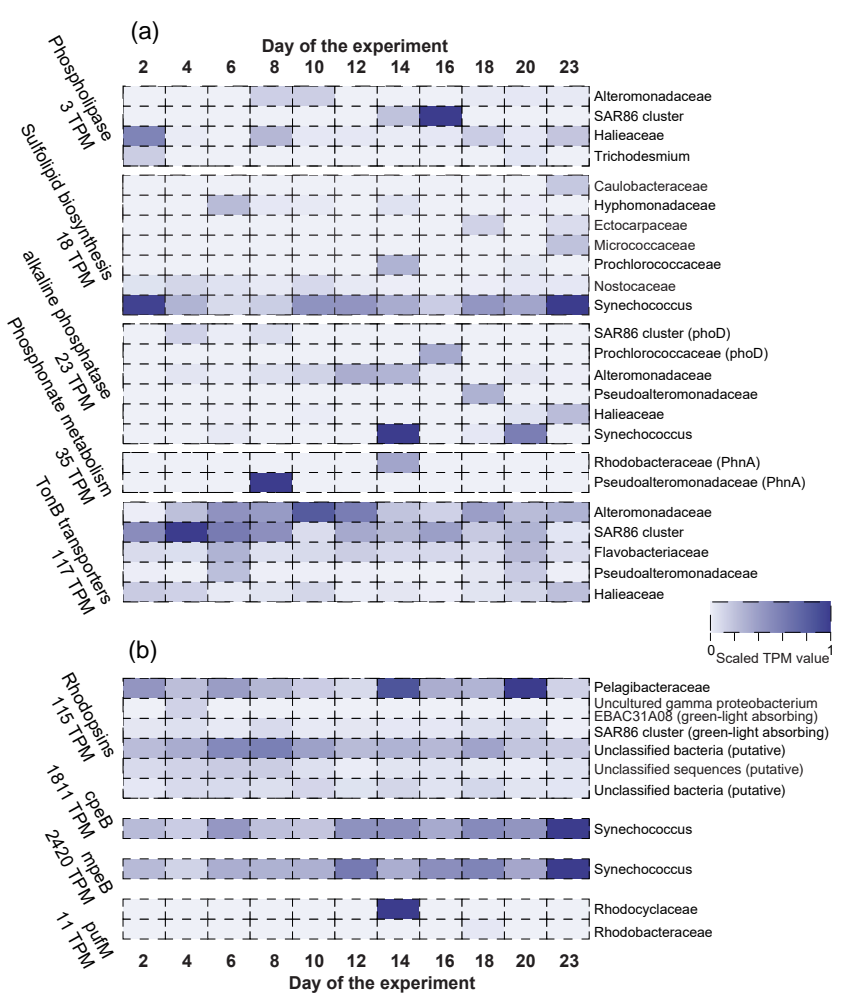

Figure 7. Heat map showing selected genes indicative for different phosphorus acquisition strategies (a) and genes for light-absorbing proteins (b). TPM counts were summed up per taxonomic family, the names of which are denoted to the right of each line. The maximum TPM value for each group is written below the name of that group. For plotting, values were scaled within each functional group, but not for each line, resulting in the maximum color density always representing the maximum TPM. After the name, in brackets, is additional annotation information, if deviant from the name of the functional group.

\subsubsection{Expression of genes involved in the assimilation of phosphate and light utilization}

In addition to nitrogen, genes involved in phosphate assimilation were analyzed in more detail. Expression of alkaline phosphatase (AP) was prominent between days 12 and 23 in different organisms (Fig. 7, alkaline phosphatase) and not expressed before the DIP fertilization, although phosphate levels were similar before the fertilization event and after day 13 (Pfreundt et al., 2016), and phosphate turnover time reached prefertilization levels after day 20 (Berthelot et al., 2015). Alteromonadaceae increased AP expression steadily from day 10 to 14 . This suggests that the microbial community was initially adapted to the ambient phosphate levels and not phosphate limited, and that the post-fertilization community had to actively acquire phosphorus (P) to fulfil their quota. In a companion paper $\mathrm{N}$ limitation, but not $\mathrm{P}$ limitation, was evident for heterotrophic bacteria throughout the experiment (Van Wambeke et al., 2016). The dom- inant photoautotroph, Synechococcus, expressed the gene for the sulfolipid biosynthesis protein, $s q d B$, in agreement with Synechococcus abundance (Fig. 7b, sulfolipid biosynthesis). Van Mooy and colleagues (Van Mooy et al., 2006, 2009) suggested that marine picocyanobacteria could minimize their $\mathrm{P}$ requirements through the synthesis of sulphoquinovosyldiacyl glycerols, for which $s q d B$ is required, and substitute phospholipids. Therefore, our finding of a high expression of the Synechococcus sqdB gene, especially towards the end of the experiment is consistent with this idea and with the increasing Synechococcus cell count towards the end of the experiment, when phosphate became limiting again (Pfreundt et al., 2016).

TonB-dependent transport allows large molecules to pass through the membrane. This strategy of exploiting larger molecules as nutrient sources is thought to be prevalent in SAR86 bacteria (Dupont et al., 2012) and indeed we found the highest expression of tonB genes for SAR86 at the beginning of the experiment, when phosphate was low. Despite increasing SAR86 cell numbers towards day 10 (Van Wambeke et al., 2016), ton $B$ expression decreased after the DIP spike, indicating a role in phosphate acquisition for the TonB transporters in SAR86. Interestingly, Halieaceae expressed ton $B$ genes together with patatin phospholipase on day 2 and weaker on day 23, indicating that phospholipids were utilized as a phosphate source prior to DIP fertilization when DIP availability was limited, and again at the end of the experiment when DIP was depleted again.

Proteorhodopsin was highly expressed, especially by SAR11 (Pelagibacter), underlining the importance of light as an additional source of ATP for this group. Bacteriorhodopsin gene expression was reported to depend on the ambient light conditions in several different bacteria, including Flavobacteria and SAR11 (Gómez-Consarnau et al., 2010; Kimura et al., 2011; Lami et al., 2009). This is consistent with our observation of upregulated proteorhodopsin expression towards the end of the experiment in the Pelagibacteraceae (Fig. 7b). There were also archaeal rhodopsins expressed, but at a $\sim 2$ orders of magnitude lower level.

\subsection{Highly expressed noncoding RNA in picocyanobacteria}

Although largely unexplored in nonmodel bacteria, ncRNAs can play important regulatory roles, e.g., in cyanobacteria in the adaptation of the photosynthetic apparatus to highlight intensities (Georg et al., 2014) or of the nitrogen assimilatory machinery to nitrogen limitation (Klähn et al., 2015). During the analysis of the 100 transcripts with the highest mean abundance, we found that 14 of these transcripts corresponded to the recently identified noncoding RNA (ncRNA) Yfr103. The Yfr103 transcripts were mapped to 14 different loci, from these 12 could be assigned to Synechococcus, 1 to Prochlorococcus, and 1 to picocyanobacteria as it was equally similar to both genera. The expression of these 
14 Yfr103 isoforms was plotted over the time course of the experiment, indicating their almost constitutive expression (Fig. S7). Yfr103 was described first as the single most abundant ncRNA in laboratory cultures of Prochlorococcus MIT9313 (Voigt et al., 2014) and here we show Yfr103 is the single most abundant transcript over the entire microbial population. These data suggest an important function of this ncRNA in marine Synechococcus and Prochlorococcus.

\section{Conclusions}

Here, we have studied how mesocosm confinement and DIP fertilization influenced transcriptional activities of the microbial community during the VAHINE experiment in the southwest Pacific. One of the most pronounced effects we observed was transcript diversification within the mesocosm, pointing to induced transcriptional responses in several taxonomic groups compared to a more stable transcript pool in the lagoon. Despite this diversification, analysis of differentially expressed transcripts amongst time points showed that global transcriptional changes roughly followed the timeline in both M1 and the lagoon. This confirms results from 16Sbased community analysis, where time was shown to be the factor most strongly influencing bacterial succession in both locations. Gene expression inside M1 was dominated by A1phaproteobacteria until day 12, with Rhodobacteraceae exhibiting a prominent peak on day 4 . This was followed by a burst in Alteromonadaceae-related gene expression on days 8 and 10 and a peak in transcript abundance from Idiomarinaceae on day 10 in rapid succession. In the lagoon, Synechococcus transcripts were the most abundant throughout the experiment, and similar abundances were reached in M1 only in P2. We further observed a tight coupling between gene expression of SAR86 and SAR11 over the whole experiment. Such coupling has been observed previously during the diel cycle (Aylward et al., 2015), whereas we have observed this phenomenon here for a longer period of time, both inside and outside (Fig. S6). Such concerted activity changes between taxonomically distinct groups should affect biogeochemical transformations and should be governed by structured ecological conditions. However, the environmental determinants driving this coupling remain to be identified.

The specific gene expression of diazotrophic cyanobacteria could be mainly attributed to Trichodesmium and Richelia intracellularis strains (diatom-diazotroph associations). UCYN-A (Candidatus Atelocyanobacterium) transcripts were the third most abundant class coming from diazotrophic cyanobacteria and declined both inside and outside after day 4, consistent with both $16 \mathrm{~S}$ - and nifH-based analyses. Transcripts related to the Epithemia turgida endosymbiont and Cyanothece ATCC 51142 increased in P2, relative to $\mathrm{P} 0$ and $\mathrm{P} 1$, consistent with the observed increase in UCYN-C nifH tags after day 14 in M1. Hence, we conclude that an unclassified relative of the Epithemia turgida endosymbiont is the main contributor to UCYN-C N2-fixing cyanobacteria.

\section{Data availability}

All raw sequencing data can be downloaded from NCBI's Sequence Read Archive (SRA) under the accession number PRJNA304389 (Pfreundt et al., 2015).

\section{The Supplement related to this article is available online at doi:10.5194/bg-13-4135-2016-supplement.}

Author contributions. Sophie Bonnet conceived and designed the experiment. Ilana Berman-Frank took part in experimental planning, preparation, and implementation. Ulrike Pfreundt, Wolfgang R. Hess, Dina Spungin, and Ilana Berman-Frank participated in experiment and sampled. Ulrike Pfreundt analyzed samples and prepared all figures. Wolfgang R. Hess and Ulrike Pfreundt wrote the manuscript and all authors contributed and revised the manuscript.

Acknowledgements. The authors thank the captain and crew of the $\mathrm{R} / \mathrm{V}$ Alis for their great support during the cruise, to Eyal Rahav throughout the experimental campaign, to Brian Haas (The Broad Institute) and Ben Fulton (Indiana University) for their patient help with the Trinity de-novo transcriptome assembler, Craig Nelson (University of Hawaii at Manoa) for his valuable advice on multivariate statistics, Björn Grüning (University of Freiburg) for his immediate actions regarding tool updates and bugs on the local Galaxy server, and Steffen Lott (University of Freiburg) for his help with the visualization tool CoVennTree. The participation of Ulrike Pfreundt, Wolfgang R. Hess, Dina Spungin, and Ilana Berman-Frank in the VAHINE experiment was supported by the German-Israeli Research Foundation (GIF), project number 1133-13.8/2011, the United States-Israel Binational Science Foundation (BSF) grant 2008048 to Ilana Berman-Frank and the metatranscriptome analysis by the EU project MaCuMBA (Marine Microorganisms: Cultivation Methods for Improving their Biotechnological Applications; grant agreement no. 311975) to Wolfgang R. Hess.

Edited by: D. G. Capone

Reviewed by: two anonymous referees

\section{References}

Aylward, F. O., Eppley, J. M., Smith, J. M., Chavez, F. P., Scholin, C. A., and DeLong, E. F.: Microbial community transcriptional networks are conserved in three domains at ocean basin scales, P. Natl. Acad. Sci. USA, 112, 5443-5448, doi:10.1073/pnas.1502883112, 2015. 
Berthelot, H., Moutin, T., L'Helguen, S., Leblanc, K., Hélias, S., Grosso, O., Leblond, N., Charrière, B., and Bonnet, S.: Dinitrogen fixation and dissolved organic nitrogen fueled primary production and particulate export during the VAHINE mesocosm experiment (New Caledonia lagoon), Biogeosciences, 12, 40994112, doi:10.5194/bg-12-4099-2015, 2015.

Bonnet, S., Berthelot, H., Turk-Kubo, K., Fawcett, S., Rahav, E., L'Helguen, S., and Berman-Frank, I.: Dynamics of $\mathrm{N}_{2}$ fixation and fate of diazotroph-derived nitrogen in a low-nutrient, low-chlorophyll ecosystem: results from the VAHINE mesocosm experiment (New Caledonia), Biogeosciences, 13, 2653-2673, doi:10.5194/bg-13-2653-2016, 2016 .

Bonnet, S., Moutin, T., Rodier, M., Grisoni, J.-M., Louis, F., Folcher, E., Bourgeois, B., Boré, J.M., and Renaud, A.: Introduction to the project VAHINE: VAriability of vertical and tropHIc transfer of diazotroph derived $\mathrm{N}$ in the south wEst Pacific, Biogeosciences, 13, 2803-2814, doi:10.5194/bg-13-28032016, 2016b.

Buchfink, B., Xie, C., and Huson, D. H.: Fast and sensitive protein alignment using DIAMOND, Nat. Methods, 12, 59-60, doi:10.1038/nmeth.3176, 2015.

Cho, J.-C. and Giovannoni, S. J.: Cultivation and growth characteristics of a diverse group of oligotrophic marine Gammaproteobacteria, Appl. Environ. Microbiol., 70, 432-440, 2004.

Dufresne, A., Ostrowski, M., Scanlan, D. J., Garczarek, L., Mazard, S., Palenik, B. P., Paulsen, I. T., de Marsac, N. T., Wincker, P., Dossat, C., Ferriera, S., Johnson, J., Post, A. F., Hess, W. R., and Partensky, F.: Unraveling the genomic mosaic of a ubiquitous genus of marine cyanobacteria, Genome Biol., 9, R90, doi:10.1186/gb-2008-9-5-r90, 2008.

Dupont, C. L., Rusch, D. B., Yooseph, S., Lombardo, M.-J., Alexander Richter, R., Valas, R., Novotny, M., Yee-Greenbaum, J., Selengut, J. D., Haft, D. H., Halpern, A. L., Lasken, R. S., Nealson, K., Friedman, R., and Craig Venter, J.: Genomic insights to SAR86, an abundant and uncultivated marine bacterial lineage, ISME J., 6, 1186-1199, doi:10.1038/ismej.2011.189, 2012.

Edgar, R. C.: Search and clustering orders of magnitude faster than BLAST, Bioinformatics, 26, 2460-2461, doi:10.1093/bioinformatics/btq461, 2010.

Forchhammer, K.: P(II) signal transducers: novel functional and structural insights, Trends Microbiol., 16, 65-72, doi:10.1016/j.tim.2007.11.004, 2008.

Foster, R. A., Kuypers, M. M. M., Vagner, T., Paerl, R. W., Musat, N., and Zehr, J. P.: Nitrogen fixation and transfer in open ocean diatom-cyanobacterial symbioses, ISME J., 5, 1484-1493, doi:10.1038/ismej.2011.26, 2011.

Frank, J. A., Lorimer, D., Youle, M., Witte, P., Craig, T., Abendroth, J., Rohwer, F., Edwards, R. A., Segall, A. M., and Burgin, A. B.: Structure and function of a cyanophage-encoded peptide deformylase, ISME J., 7, 1150-1160, doi:10.1038/ismej.2013.4, 2013.

Frias-Lopez, J., Shi, Y., Tyson, G. W., Coleman, M. L., Schuster, S. C., Chisholm, S. W., and Delong, E. F.: Microbial community gene expression in ocean surface waters, P. Natl. Acad. Sci. USA, 105, 3805-3810, doi:10.1073/pnas.0708897105, 2008.

Ganesh, S., Bristow, L. A., Larsen, M., Sarode, N., Thamdrup, B. and Stewart, F. J.: Size-fraction partitioning of community gene transcription and nitrogen metabolism in a marine oxygen mini- mum zone, ISME J., 9, 2682-2696, doi:10.1038/ismej.2015.44, 2015.

García-Martínez, J., Acinas, S. G., Massana, R., and RodríguezValera, F.: Prevalence and microdiversity of Alteromonas macleodii-like microorganisms in different oceanic regions, Environ. Microbiol., 4, 42-50, 2002.

Georg, J., Dienst, D., Schürgers, N., Wallner, T., Kopp, D., Stazic, D., Kuchmina, E., Klähn, S., Lokstein, H., Hess, W. R., and Wilde, A.: The Small Regulatory RNA SyR1/PsrR1 Controls Photosynthetic Functions in Cyanobacteria, Plant Cell Online, 26, 3661-3679, doi:10.1105/tpc.114.129767, 2014.

Gifford, S. M., Sharma, S., and Moran, M. A.: Linking activity and function to ecosystem dynamics in a coastal bacterioplankton community, Front. Microbiol., 5, 185, doi:10.3389/fmicb.2014.00185, 2014.

Gómez-Consarnau, L., Akram, N., Lindell, K., Pedersen, A., Neutze, R., Milton, D. L., González, J. M., and Pinhassi, J.: Proteorhodopsin phototrophy promotes survival of marine bacteria during starvation, PLoS Biol., 8, e1000358, doi:10.1371/journal.pbio.1000358, 2010.

Haas, B. J., Papanicolaou, A., Yassour, M., Grabherr, M., Blood, P. D., Bowden, J., Couger, M. B., Eccles, D., Li, B., Lieber, M., Macmanes, M. D., Ott, M., Orvis, J., Pochet, N., Strozzi, F., Weeks, N., Westerman, R., William, T., Dewey, C. N., Henschel, R., Leduc, R. D., Friedman, N., and Regev, A.: De novo transcript sequence reconstruction from RNA-seq using the Trinity platform for reference generation and analysis, Nat. Protoc., 8, 1494-1512, doi:10.1038/nprot.2013.084, 2013.

Hewson, I., Poretsky, R. S., Tripp, H. J., Montoya, J. P., and Zehr, J. P.: Spatial patterns and light-driven variation of microbial population gene expression in surface waters of the oligotrophic open ocean, Environ. Microbiol., 12, 1940-1956, doi:10.1111/j.14622920.2010.02198.x, 2010.

Hilton, J. A., Satinsky, B. M., Doherty, M., Zielinski, B., and Zehr, J. P.: Metatranscriptomics of $\mathrm{N}_{2}$-fixing cyanobacteria in the Amazon River plume, ISME J., 9, 1557-1569, doi:10.1038/ismej.2014.240, 2015.

Huergo, L. F., Chandra, G., and Merrick, M.: P(II) signal transduction proteins: nitrogen regulation and beyond, FEMS Microbiol. Rev., 37, 251-283, doi:10.1111/j.1574-6976.2012.00351.x, 2013.

Hunt, D. E., Lin, Y., Church, M. J., Karl, D. M., Tringe, S. G., Izzo, L. K., and Johnson, Z. I.: Relationship between Abundance and Specific Activity of Bacterioplankton in Open Ocean Surface Waters, Appl. Environ. Microbiol., 79, 177-184, doi:10.1128/AEM.02155-12, 2013.

Huson, D. H. and Weber, N.: Microbial community analysis using MEGAN, Methods Enzymol., 531, 465-485, doi:10.1016/B9780-12-407863-5.00021-6, 2013.

Ivars-Martinez, E., Martin-Cuadrado, A.-B., D’Auria, G., Mira, A., Ferriera, S., Johnson, J., Friedman, R., and Rodriguez-Valera, F.: Comparative genomics of two ecotypes of the marine planktonic copiotroph Alteromonas macleodii suggests alternative lifestyles associated with different kinds of particulate organic matter, ISME J., 2, 1194-1212, doi:10.1038/ismej.2008.74, 2008.

Jones, D. S., Flood, B. E., and Bailey, J. V.: Metatranscriptomic insights into polyphosphate metabolism in marine sediments, ISME J., 10, 1015-1019, doi:10.1038/ismej.2015.169, 2015. 
Kimura, H., Young, C. R., Martinez, A., and Delong, E. F.: Light-induced transcriptional responses associated with proteorhodopsin-enhanced growth in a marine flavobacterium, ISME J., 5, 1641-1651, doi:10.1038/ismej.2011.36, 2011.

Klähn, S., Schaal, C., Georg, J., Baumgartner, D., Knippen, G., Hagemann, M., Muro-Pastor, A. M., and Hess, W. R.: The sRNA NsiR4 is involved in nitrogen assimilation control in cyanobacteria by targeting glutamine synthetase inactivating factor IF7, P. Natl. Acad. Sci. USA, 112, E6243-6252, doi:10.1073/pnas.1508412112, 2015.

Knapp, A. N., Fawcett, S. E., Martínez-Garcia, A., Leblond, N., Moutin, T., and Bonnet, S.: Nitrogen isotopic evidence for a shift from nitrate- to diazotroph-fueled export production in VAHINE mesocosm experiments, Biogeosciences Discuss., 12, 1990119939, doi:10.5194/bgd-12-19901-2015, 2015.

Kopylova, E., Noé, L., and Touzet, H.: SortMeRNA: fast and accurate filtering of ribosomal RNAs in metatranscriptomic data, Bioinforma. Oxf. Engl., 28, 3211-3217, doi:10.1093/bioinformatics/bts611, 2012.

Lami, R., Cottrell, M. T., Campbell, B. J., and Kirchman, D. L.: Light-dependent growth and proteorhodopsin expression by Flavobacteria and SAR11 in experiments with Delaware coastal waters, Environ. Microbiol., 11, 3201-3209, doi:10.1111/j.14622920.2009.02028.x, 2009.

Langmead, B.: Aligning short sequencing reads with Bowtie, Curr. Protoc. Bioinforma. Ed. Board Andreas Baxevanis Al, Chapter 11, Unit 11.7, doi:10.1002/0471250953.bi1107s32, 2010.

Leblanc, K., Cornet, V., Caffin, M., Rodier, M., Desnues, A., Berthelot, H., Turk-Kubo, K., and Heliou, J.: Phytoplankton community structure in the VAHINE MESOCOSM experiment, Biogeosciences Discuss., doi:10.5194/bg-2015-605, in review, 2016.

Li, B. and Dewey, C. N.: RSEM: accurate transcript quantification from RNA-Seq data with or without a reference genome, BMC Bioinformatics, 12, 323, doi:10.1186/1471-2105-12-323, 2011.

Lindell, D. and Post, A. F.: Ecological aspects of ntcA gene expression and its use as an indicator of the nitrogen status of marine Synechococcus spp, Appl. Environ. Microbiol., 67, 3340-3349, doi:10.1128/AEM.67.8.3340-3349.2001, 2001.

López-Pérez, M., Gonzaga, A., Martin-Cuadrado, A.-B., Onyshchenko, O., Ghavidel, A., Ghai, R., and Rodriguez-Valera, F.: Genomes of surface isolates of Alteromonas macleodii: the life of a widespread marine opportunistic copiotroph, Sci. Rep., 2, 696, doi:10.1038/srep00696, 2012.

Lott, S. C., Voß, B., Hess, W. R., and Steglich, C.: CoVennTree: a new method for the comparative analysis of large datasets, Front. Genet., 6, 43, doi:10.3389/fgene.2015.00043, 2015.

Luo, Y.-W., Doney, S. C., Anderson, L. A., Benavides, M., BermanFrank, I., Bode, A., Bonnet, S., Boström, K. H., Böttjer, D., Capone, D. G., Carpenter, E. J., Chen, Y. L., Church, M. J., Dore, J. E., Falcón, L. I., Fernández, A., Foster, R. A., Furuya, K., Gómez, F., Gundersen, K., Hynes, A. M., Karl, D. M., Kitajima, S., Langlois, R. J., LaRoche, J., Letelier, R. M., Marañón, E., McGillicuddy Jr., D. J., Moisander, P. H., Moore, C. M., Mouriño-Carballido, B., Mulholland, M. R., Needoba, J. A., Orcutt, K. M., Poulton, A. J., Rahav, E., Raimbault, P., Rees, A. P., Riemann, L., Shiozaki, T., Subramaniam, A., Tyrrell, T., TurkKubo, K. A., Varela, M., Villareal, T. A., Webb, E. A., White, A. E., Wu, J., and Zehr, J. P.: Database of diazotrophs in global ocean: abundance, biomass and nitrogen fixation rates, Earth Syst. Sci. Data, 4, 47-73, doi:10.5194/essd-4-47-2012, 2012.

Ma, Y., Allen, L. Z., and Palenik, B.: Diversity and genome dynamics of marine cyanophages using metagenomic analyses, Environ. Microbiol. Rep., 6, 583-594, 2014.

Markowitz, V. M., Chen, I.-M. A., Chu, K., Pati, A., Ivanova, N. N., and Kyrpides, N. C.: Ten Years of Maintaining and Expanding a Microbial Genome and Metagenome Analysis System, Trends Microbiol., doi:10.1016/j.tim.2015.07.012, 2015.

Martin-Cuadrado, A.-B., Garcia-Heredia, I., Moltó, A. G., LópezÚbeda, R., Kimes, N., López-García, P., Moreira, D., and Rodriguez-Valera, F.: A new class of marine Euryarchaeota group II from the Mediterranean deep chlorophyll maximum, ISME J., 9, 1619-1634, doi:10.1038/ismej.2014.249, 2015.

Moore, R. B., Oborník, M., Janouskovec, J., Chrudimský, T., Vancová, M., Green, D. H., Wright, S. W., Davies, N. W., Bolch, C. J. S., Heimann, K., Slapeta, J., Hoegh-Guldberg, O., Logsdon, J. M., and Carter, D. A.: A photosynthetic alveolate closely related to apicomplexan parasites, Nature, 451, 959-963, doi:10.1038/nature06635, 2008.

Moran, M. A., Satinsky, B., Gifford, S. M., Luo, H., Rivers, A., Chan, L.-K., Meng, J., Durham, B. P., Shen, C., Varaljay, V. A., Smith, C. B., Yager, P. L., and Hopkinson, B. M.: Sizing up metatranscriptomics, ISME J., 7, 237-243, doi:10.1038/ismej.2012.94, 2013.

Nakayama, T., Kamikawa, R., Tanifuji, G., Kashiyama, Y., Ohkouchi, N., Archibald, J. M., and Inagaki, Y.: Complete genome of a nonphotosynthetic cyanobacterium in a diatom reveals recent adaptations to an intracellular lifestyle, P. Natl. Acad. Sci. USA, 111, 11407-11412, doi:10.1073/pnas.1405222111, 2014.

Oborník, M., Modrý, D., Lukeš, M., Cernotíková-Stř̌́brná, E., Cihlář, J., Tesařová, M., Kotabová, E., Vancová, M., Prášil, O., and Lukeš, J.: Morphology, ultrastructure and life cycle of Vitrella brassicaformis n. sp., n. gen., a novel chromerid from the Great Barrier Reef, Protist, 163, 306-323, doi:10.1016/j.protis.2011.09.001, 2012.

Oksanen, J., Blanchet, F. G., Kindt, R., Legendre, P., Minchin, P. R., O’Hara, R. B., Simpson, G. L., Solymos, P., Stevens, M. H. H., and Wagner, H.: vegan: Community Ecology Package, available at: http://CRAN.R-project.org/package=vegan (last access: 7 February 2016), 2015.

Pfreundt, U., Miller, D., Adusumilli, L., Stambler, N., BermanFrank, I., and Hess, W. R.: Depth dependent metatranscriptomes of the marine pico-/nanoplanktonic communities in the Gulf of Aqaba/Eilat during seasonal deep mixing, Mar. Genomics, $18 \mathrm{Pt}$ B, 93-95, doi:10.1016/j.margen.2014.06.005, 2014.

Pfreundt, U., Spungin, D., Berman-Frank, I., Bonnet, S., and Hess, W. R.: 16S rRNA gene amplicon and metatranscriptomic datasets from the VAHINE mesocosm experiment in the New Caledonian Lagoon, South West Pacific, http://www.ncbi.nlm.nih.gov/ bioproject/PRJNA304389/, 2015.

Pfreundt, U., Van Wambeke, F., Caffin, M., Bonnet, S., and Hess, W. R.: Succession within the prokaryotic communities during the VAHINE mesocosms experiment in the New Caledonia lagoon, Biogeosciences, 13, 2319-2337, doi:10.5194/bg-13-2319-2016, 2016.

Pinto, F. L., Thapper, A., Sontheim, W., and Lindblad, P.: Analysis of current and alternative phenol based RNA extraction 
methodologies for cyanobacteria, BMC Mol. Biol., 10, 79, doi:10.1186/1471-2199-10-79, 2009.

Poretsky, R. S., Hewson, I., Sun, S., Allen, A. E., Zehr, J. P., and Moran, M. A.: Comparative day/night metatranscriptomic analysis of microbial communities in the North Pacific subtropical gyre, Environ. Microbiol., 11, 1358-1375, doi:10.1111/j.14622920.2008.01863.x, 2009.

Prechtl, J., Kneip, C., Lockhart, P., Wenderoth, K., and Maier, U.-G.: Intracellular spheroid bodies of rhopalodia gibba have nitrogen-fixing apparatus of cyanobacterial origin, Mol. Biol. Evol., 21, 1477-1481, doi:10.1093/molbev/msh086, 2004.

Robinson, M. D., McCarthy, D. J., and Smyth, G. K.: edgeR: a Bioconductor package for differential expression analysis of digital gene expression data, Bioinformatics, 26, 139-140, doi:10.1093/bioinformatics/btp616, 2010.

Scanlan, D. J., Ostrowski, M., Mazard, S., Dufresne, A., Garczarek, L., Hess, W. R., Post, A. F., Hagemann, M., Paulsen, I., and Partensky, F.: Ecological genomics of marine picocyanobacteria, Microbiol. Mol. Biol. Rev., 73, 249-299, doi:10.1128/MMBR.00035-08, 2009.

Schäfer, H., Servais, P., and Muyzer, G.: Successional changes in the genetic diversity of a marine bacterial assemblage during confinement, Arch. Microbiol., 173, 138-145, 2000.

Shi, Y., Tyson, G. W., and DeLong, E. F.: Metatranscriptomics reveals unique microbial small RNAs in the ocean's water column, Nature, 459, 266-269, doi:10.1038/nature08055, 2009.

Spring, S., Riedel, T., Spröer, C., Yan, S., Harder, J., and Fuchs, B. M.: Taxonomy and evolution of bacteriochlorophyll a-containing members of the OM60/NOR5 clade of marine gammaproteobacteria: description of Luminiphilus syltensis gen. nov., sp. nov., reclassification of Haliea rubra as Pseudohaliea rubra gen. nov., comb. nov., and emendation of Chromatocurvus halotolerans, BMC Microbiol., 13, 118, doi:10.1186/1471-2180-13-118, 2013.

Steglich, C., Stazic, D., Lott, S. C., Voigt, K., Greengrass, E., Lindell, D., and Hess, W. R.: Dataset for metatranscriptome analysis of Prochlorococcus-rich marine picoplankton communities in the Gulf of Aqaba, Red Sea, Mar. Genomics, 19, 5-7, 2015.
Tolonen, A. C., Aach, J., Lindell, D., Johnson, Z. I., Rector, T., Steen, R., Church, G. M., and Chisholm, S. W.: Global gene expression of Prochlorococcus ecotypes in response to changes in nitrogen availability, Mol. Syst. Biol., 2, 53, doi:10.1038/msb4100087, 2006.

Turk-Kubo, K. A., Frank, I. E., Hogan, M. E., Desnues, A., Bonnet, S., and Zehr, J. P.: Diazotroph community succession during the VAHINE mesocosm experiment (New Caledonia lagoon), Biogeosciences, 12, 7435-7452, doi:10.5194/bg-12-7435-2015, 2015.

Van Mooy, B. A. S., Rocap, G., Fredricks, H. F., Evans, C. T. and Devol, A. H.: Sulfolipids dramatically decrease phosphorus demand by picocyanobacteria in oligotrophic marine environments, P. Natl. Acad. Sci. USA, 103, 8607-8612, doi:10.1073/pnas.0600540103, 2006.

Van Mooy, B. A. S., Fredricks, H. F., Pedler, B. E., Dyhrman, S. T., Karl, D. M., Koblízek, M., Lomas, M. W., Mincer, T. J., Moore, L. R., Moutin, T., Rappé, M. S., and Webb, E. A.: Phytoplankton in the ocean use non-phosphorus lipids in response to phosphorus scarcity, Nature, 458, 69-72, doi:10.1038/nature07659, 2009.

Van Wambeke, F., Pfreundt, U., Barani, A., Berthelot, H., Moutin, T., Rodier, M., Hess, W. R., and Bonnet, S.: Heterotrophic bacterial production and metabolic balance during the VAHINE mesocosm experiment in the New Caledonia lagoon, Biogeosciences, 13, 3187-3202, doi:10.5194/bg-13-3187-2016, 2016.

Voigt, K., Sharma, C. M., Mitschke, J., Joke Lambrecht, S., Voß, B., Hess, W. R., and Steglich, C.: Comparative transcriptomics of two environmentally relevant cyanobacteria reveals unexpected transcriptome diversity, ISME J., 8, 2056-2068, doi:10.1038/ismej.2014.57, 2014.

Wemheuer, B., Wemheuer, F., Hollensteiner, J., Meyer, F.-D., Voget, S., and Daniel, R.: The green impact: bacterioplankton response toward a phytoplankton spring bloom in the southern North Sea assessed by comparative metagenomic and metatranscriptomic approaches, Front. Microbiol., 6, 805, doi:10.3389/fmicb.2015.00805, 2015. 